\title{
THE RISE AND FALL OF THE VECTOR EPSILON ALGORITHM
}

\author{
by \\ I.D. Coope \\ Department of Mathematics, University of Canterbury, \\ Christchurch, New Zealand. \\ P.R. Graves-Morris \\ Department of Mathematics, University of Bradford, \\ Bradford, West Yorkshire, England BD7 1DP.
}

No. 71

November, 1992

Submitted to Numerical Algorithms, Oxford, August 1992. 


\title{
THE RISE AND FALL OF THE VECTOR EPSILON ALGORITHM
}

\author{
I.D. Coope \\ Department of Mathematics \\ University of Canterbury \\ Christchurch \\ New Zealand.
}

\author{
P.R. Graves-Morris \\ Department of Mathematics \\ University of Bradford \\ Bradford, West Yorkshire \\ England BD7 1DP.
}

\begin{abstract}
Summary. The performance of the vector epsilon is governed by two important mathematical theorems which are briefly reviewed in context. We note that the performance of the vector epsilon algorithm is inevitably qualitatively incorrect for sequences whose generating functions have poles near unity. This difficulty is avoided by the use of hybrid vector Padé approximants.
\end{abstract}

\section{Introduction}

In Section 2 of this paper, we briefly describe the vector epsilon algorithm. Results which give support to the claim that the vector epsilon algorithm should be regarded as a useful and, in context, reliable method for the acceleration of vector sequences are stated. We conclude Section 2 with an account of how the vector epsilon algorithm is generalised to become the functional epsilon algorithm.

In Section 3, we describe a drawback to the use of the vector epsilon algorithm. We show that its performance for sequences arising near poles of their underlying generating function is not what one would naïvely expect or want.

In Section 4, we discuss the performance of a modified version of the vector epsilon algorithm, called the hybrid vector epsilon algorithm. Although this algorithm is not, as yet, iterative, it does behave satisfactorily near poles of the underlying generating function, and it has been found to be remarkably accurate on test examples. An example of use of the hybrid functional Padé approximant in the context of iterative solution of a simple integral equation is given. 


\section{The rise of the vector epsilon algorithm}

The epsilon algorithm is primarily an algorithm for the acceleration of the convergence of a given sequence of numbers. It originated from the work of Shanks [1955] and Wynn [1956]. It is described by Baker [1974, Chap. 6], Baker \& Graves-Morris [1981, Chap. 3] and Brezinski [1980, Chap, 3] for example. If the given sequence is a sequence of vectors, then its convergence can similarly be accelerated using the vector epsilon algorithm [Wynn, 1962, 1963].

In this paper we suppose that a sequence of vectors is given. The formalism works equally well for a sequence of functions regarded as elements of a vector space, and we will include this case explicitly later. For the moment, suppose that a sequence $S$ of real $d$-dimensional vectors is given:

$$
S:=\left\{\boldsymbol{s}_{i}, i=0,1,2, \cdots: \boldsymbol{s}_{\boldsymbol{i}} \in \mathbf{R}^{d}\right\}
$$

The vector $\varepsilon$-algorithm is initialised with

$$
\begin{gathered}
\varepsilon_{-1}^{(j)}=0, j=1,2,3, \ldots \\
\varepsilon_{0}^{(j)}=s_{j}, j=0,1,2, \ldots
\end{gathered}
$$

The remainder of the vector $\epsilon$-table is calculated recursively by using

$$
\varepsilon_{k+1}^{(j)}=\varepsilon_{k-1}^{(j+1)}+\left[\varepsilon_{k}^{(j+1)}-\varepsilon_{k}^{(j)}\right]^{-1}
$$

for $k=1,2,3, \ldots$, for $j=0,1,2, \ldots$, provided the desired inverses exist. Reciprocals of vectors are defined as their Moore-Penrose inverses:

$$
\boldsymbol{v}^{-1}:=\boldsymbol{v} /|\boldsymbol{v}|^{2}, \quad \boldsymbol{v} \in \mathbf{R}^{d}
$$

In this paper, we restrict attention to real vectors, and omit the generalisation to complex-valued quantities. Associated with the entry $\varepsilon_{2 k}^{(j)}$ in an even index column of the vector $\varepsilon$-table is the vector Padé approximant of type $[n / 2 k]$, where $n=j+2 k$. This approximant takes the rational form

$$
\boldsymbol{r}^{[n / 2 k]}(z)=\boldsymbol{p}^{[n / 2 k]}(z) / q^{[n / 2 k]}(z)
$$

where $q^{[n / 2 k]}(z)$ is a polynomial of degree $2 k$ and $p^{[n / 2 k]}(z)$ is a vector polynomial of degree $n$. In this paper, we are ignoring all cases of degeneracy and degree deficiency for ease of presentation. These matters, as well as those of the specification, existence and construction of $\boldsymbol{p}^{[n / 2 k]}(z)$ and 
$q^{[n / 2 k]}(z)$ are treated by Graves-Morris \& Jenkins [1989]. For present purposes, we may assume that the denominator is given by

$$
q^{[n / 2 k]}(z)=\left|\begin{array}{ccccc}
0 & M_{01} & \ldots & M_{0,2 k-1} & M_{0,2 k} \\
-M_{01} & 0 & \ldots & M_{1,2 k-1} & M_{1,2 k} \\
\vdots & \vdots & & \vdots & \vdots \\
-M_{0,2 k-1} & -M_{1,2 k-1} & \ldots & 0 & M_{2 k-1,2 k} \\
z^{2 k} & z^{2 k-1} & \ldots & z & 1
\end{array}\right|
$$

where

$$
M_{i j}:=\sum_{l=0}^{j-i-1} c_{l+i+n-2 k+1} \cdot c_{j-l+n-2 k}, \quad j>i .
$$

(In practice, one might well obtain $q^{[n / 2 k]}(z)$ coefficientwise using a linear equation solver for the set of linear equations underlying (2.5)). The numerator is obtained from (2.4) and

$$
\boldsymbol{p}^{[n / 2 k]}(z):=\left[\boldsymbol{f}(z) q^{[n / 2 k]}(z)\right]_{0}^{n}
$$

where the notation $[\phi(z)]_{0}^{n}$ indicates that the first $n+1$ terms of the Maclaurin series of $\phi(z)$ are formed. The function $f(z)$ in (2.7) is the generating function of the given sequence. To form it, we define

$$
c_{0}:=s_{0}, \quad c_{i}:=\Delta s_{i-1}:=s_{i}-s_{i-1}, \quad i=1,2,3, \ldots
$$

and then $f(z)$ is at least formally defined by

$$
f(z)=\sum_{i=0}^{\infty} c_{i} z^{i}
$$

The association between the vector Padé approximant $\boldsymbol{r}^{[n / 2 k]}(z)$ and the entry $\varepsilon_{2 k}^{(j)}$ in the vector epsilon table is [Graves-Morris, 1983]

$$
\varepsilon_{2 k}^{(j)}=\boldsymbol{r}^{[n / 2 k]}(1)
$$

with $j:=n-2 k$. Again, we re-emphasise that the phrase "except in cases of degeneracy" is to be understood as applicable throughout this paper.

To understand (2.4) and (2.5) better, it is helpful to consider the reduction to the scalar case as the one-dimensional vector case. Then $\boldsymbol{r}^{[n / 2 k]}(z)$ defined by $(2.4)$ equals the ordinary Padé approximant $r^{[n-k / k]}(z)$ of type $[n-k / k]$ for $f(z) \equiv f(z)$, where $d=1$. The equality (2.10) holds in the form

$$
\varepsilon_{2 k}^{(j)}=\boldsymbol{r}^{[n-k / k]}(1)
$$


for this self-same Padé approximant. We understand these results in terms of a factorisation of $q^{[n / 2 k]}(z)$ and cancellation of a common factor of degree $k$ between $\boldsymbol{p}^{[n / 2 k]}(z)$ and $q^{[n / 2 k]}(z)$ in the case when $d=1$.

The potential performance of the vector epsilon algorithm became clear from the following theorem. It is McLeod's [1971] theorem, and it gave the first boost towards the more widespread acceptance of the vector epsilon algorithm.

Theorem 2.1 Suppose that the vector sequence $S$ of (2.1) satisfies a non-trivial recurrence relation

$$
\sum_{i=0}^{k} \beta_{i} \boldsymbol{s}_{i+j}=\left[\sum_{i=0}^{k} \beta_{i}\right] \boldsymbol{a}, \quad j=0,1,2, \ldots .
$$

Then the vector $\varepsilon$-algorithm (2.2) leads to

$$
\varepsilon_{2 k}^{(j)}=a
$$

provided that zero divisors are not encountered in the construction.

A simplified corollary of this theorem states that if the vector epsilon algorithm is applied to a given sequence having precisely $k$ distinct geometric components and its limiting value is $\boldsymbol{s}_{\infty}$, then the entries in the column of index $2 k$ are identical to $a$ : i.e. $\varepsilon_{2 k}^{(j)}=a=s_{\infty}$.

An excellent review of the performance of a number of algorithms on some new and some standard problems was given by Smith, Ford and Sidi [1987]. In general terms, they concluded that the performance of the vector epsilon algorithm is comparable to, if not better than, any other single general purpose vector accelerator, bearing in mind that, for each algorithm they considered, examples of poor performance can be found. We regard the conclusions of this survey paper as the second boost for the vector epsilon algorithm.

Finally, and continuing to omit details associated with degeneracy, we cite the following theorem which is based on that of Graves-Morris and Saff [1988]. Convergence of the column of index $2 k$ of the vector epsilon table is implicitly governed by this theorem.

Theorem 2.2 Let

$$
f(z)=\boldsymbol{g}(z) / Q(z)
$$

be analytic in $|z|<\rho$, excepting only at the real points $\left\{x_{i}\right\}_{i=1}^{k}$ which are the zeros of

$$
Q(z)=\prod_{i=1}^{k}\left(z-x_{i}\right)
$$

Let $z$ be a point in the annulus

$$
\max _{1 \leq i \leq k}\left|x_{i}\right|<z<\rho
$$


Then

$$
\lim _{n \rightarrow \infty} p^{[n / 2 k]}(z) / q^{[n / 2 k]}(z)=f(z)
$$

Let $\dot{q}^{[n / 2 k]}$ be the leading coefficient of $q^{[n / 2 k]}(z)$, so that

$$
Q_{n}(z):=q^{[n / 2 k]} / \dot{q}^{[n / 2 k]}
$$

is the monic denominator polynomial. Then these polynomials converge:

$$
\lim _{n \rightarrow \infty} Q_{n}(z)=Q(z)^{2}
$$

Again a simplified version of this theorem states that the column $\varepsilon_{2 k}^{(j)}$ of the vector $\varepsilon$-table converges when the given vector sequence $S_{n}$ has precisely $k$ distinct, dominant real components and some analytic background. This result is a natural sequel to McLeod's theorem.

An application of these results has been found in the acceleration of the convergence of a sequence arising from SOR iteration. Using relativeley standard terminology [Varga, 1962], the solution of the system

$$
A \boldsymbol{x}=\boldsymbol{b}
$$

may be based on the decomposition

$$
A=D-L-U
$$

and the use of the iteration matrix

$$
E=(D-\omega L)^{-1}\{(1-\omega) D+\omega U\}
$$

By using a value of $\omega$ designed to ensure that $E$ has precisely one dominant eigenvalue and corresponding eigenvector, the column $\varepsilon_{2}^{(j)}$ of the vector epsilon algorithm has very favourable convergence properties [Graves-Morris, 1992].

As the previous example indicates, most interest arising from practical applications centres on the performance of the vector epsilon algorithm for cases in which $d$, the dimension of the vectors, is large. In fact, all the previous results have analogues in the case $d=\infty$. This topic is called functional Padé approximation, and the convergence of sequences of functions may be accelerated by the use of the functional $\varepsilon$-algorithm [Brezinski, 1975]. For the case of $f(x) \in L_{2}(a, b) \cap \mathbf{R}$, we define the "inverse"

$$
[f(x)]^{-1}=f(x) / \int_{a}^{b}|f(x)|^{2} d x
$$


for use in the equation (2.2). With this definition, which presupposes some natural specification of $(a, b)$, or indeed any other appropriate inner product norm, the functional epsilon algorithm can be implemented. The columns of even index in the epsilon table derived are sequences of functions which are intended to converge faster than the given sequence.

Analogously to (2.4),

$$
r^{[n / 2 k]}(x, \lambda)=p^{[n / 2 k]}(x, \lambda) / q^{[n / 2 k]}(\lambda)
$$

is the form of the functional Padé approximant of type $[n / 2 k]$ for a given series

$$
f(x, \lambda)=\sum_{i=0}^{\infty} c_{i}(x) \lambda^{i}
$$

The denominator polynomial $q^{[n / 2 k]}(\lambda)$ is given by the same formula $(2.5)$, but with

$$
M_{i j}:=\sum_{l=0}^{j-i-1} \int_{a}^{b} c_{l+i+n-2 k+1}(x) c_{j-l+n-2 k}(x) d x, \quad j>i .
$$

replacing (2.6). The numerator $p^{[n / 2 k]}(x, \lambda)$ is a polynomial in $\lambda$ of degree $n$ and it lies in $L_{2}(a, b)$ as a function of $x$; it is given by

$$
p^{[n / 2 k]}(x, \lambda):=\left[f(x, \lambda) q^{[n / 2 k]}(\lambda)\right]_{0}^{n}
$$

analogously to (2.7). The association between functional Padé approximants and the entries in the functional epsilon table is given by

$$
\varepsilon_{2 k}^{(j)}(x)=r^{[n / 2 k]}(x, \lambda)
$$

analogously to (2.10).

Both Theorems 2.1 and 2.2 have natural extensions to the case of functional Padé approximation with similar proofs. In the reverse direction, results for the case of vector Padé approximants for $\left\{\boldsymbol{s}_{i}: \boldsymbol{s}_{i} \in \mathbf{R}^{d}\right\}$ follow from those for functional Padé approximants for $\left\{s_{i}(x): s_{i}(x) \in L_{2}(a, b)\right\}$ using functions $s_{i}(x)$ which are piecewise constant on $[0, d)$. Explicitly, the connection is made with

$$
s_{i}(x):=\left(s_{i}\right)_{j}, \quad \text { where } j:=[x+1]
$$

and $[\cdot]$ denotes the integer part, so that the integer $j \in[1, d]$. 


\section{The fall of the vector epsilon algorithm}

In this section, we describe a drawback to the vector epsilon algorithm, compared to its scalar counterpart, which does not appear to have been explicitly recognised as such hitherto.

It concerns the situation when the given series is nearly divergent, or, to be more general and more precise, when the underlying generating function has a pole close to unity. In the scalar case, we would have convergence in the chordal metric, which is, at least qualitatively, satisfactory. Again, we consider standard, ideal conditions under which the column $\varepsilon_{2 k}^{(j)}$ converges, corresponding to those of Theorem 2.2. Let the given initial sequence be

$$
\left\{\varepsilon_{0}^{(j)}\right\}_{j=0}^{\infty}=\left\{s_{0}, s_{1}, s_{2}, \cdots: s_{i} \in \mathbf{R}^{d}\right\}
$$

We take $c_{0}:=s_{0}$, and form the differences

$$
c_{j+1}:=s_{j+1}-s_{j}=\Delta s_{j}, \quad j=0,1,2, \ldots
$$

and the associated generating function

$$
\boldsymbol{f}(z)=\sum_{j=0}^{\infty} \boldsymbol{c}_{j} z^{j}
$$

as in (2.9). We suppose that $\boldsymbol{f}(z)$ is analytic at $z=1$, and that it has a nearby pole at $z=1+\eta$ for $|\eta| \ll 1, \quad \eta \in \mathbf{R}$. We suppose that $\boldsymbol{f}(z)$ has $k$ poles within $|z|<1$, and satisfies the other conditions of Theorem 2.2, or, more precisely, those of the theorem of Graves-Morris and Saff [1988]. Then (2.13) implies that

$$
\lim _{j \rightarrow \infty} \varepsilon_{2 k}^{(j)}=f(1)
$$

i.e. the column of index $2 k$ converges.

The principal point we wish to make is that the result (3.3) is true mathematically, but is misleading from a numerical point of view. The source of the difficulty is not rounding error, which is a serious drawback to the use of the epsilon algorithm, but approximation error. Beginning with (2.14), which is well borne out numerically, we see that the zeros of $q^{[n / 2 k]}(z)$ tend to a double zero at $z=1+\eta$.

A consequence of the factorisation condition

$$
\left.q^{[n / 2 k]}(x)|| \boldsymbol{p}^{[n / 2 k]}(x)\right|^{2}, \quad x \in \mathbf{R}
$$

[Graves-Morris, 1983] in this situation is that

$$
\lim _{n \rightarrow \infty} \boldsymbol{p}^{[n / 2 k]}(1+\eta)=0 .
$$


In practice, this means that $\boldsymbol{p}^{[n / 2 k]}(z) \cdot \boldsymbol{p}^{*[n / 2 k]}(z)$ has a pair of complex conjugate zeros near $1+\eta$. To visualise the consequences and implications of these results, we give the following illustrative numerical example. We consider the performance of the functional epsilon algorithm, and view it as the $\mathbf{R}^{\infty}$ limit of the foregoing analysis.

The initialising sequence is taken as the iterative solution of the linear integral equation

$$
f(x, \lambda)=1+\lambda \int_{0}^{1}\{1+|x-y|\} f(y, \lambda) d y
$$

previously considered by Graves-Morris [1990]. The first few terms are

$$
\begin{aligned}
f(x, \lambda) & =\sum_{i=0}^{\infty} c_{i}(x) \lambda^{i} \\
& =1+\left[\frac{5}{4}+\left(x-\frac{1}{2}\right)^{2}\right] \lambda+\left[\frac{161}{96}+\frac{5}{4}\left(x-\frac{1}{2}\right)^{2}+\frac{1}{6}\left(x-\frac{1}{2}\right)^{4}\right] \lambda^{2}+\ldots
\end{aligned}
$$

Using (2.17)-(2.19), we can form the functional Padé approximants to this series. The integral equation (3.6) is interesting as a Fredholm integral equation of the second kind possessing a non-degenerate kernel and an explicit solution, namely

$$
f(x, \lambda)=\frac{2 \cosh \nu\left(x-\frac{1}{2}\right)}{2 \cosh (\nu / 2)-3 \nu \sinh (\nu / 2)}
$$

where $\nu=\sqrt{2 \lambda}$. The denominator of (3.8) is analytic as a function of $\lambda$ and has just one simple zero at $\nu_{c}=1.22290658 \ldots$, i.e. $\lambda_{c}=0.7477502556$.

We use the sequences associated with (3.7) as our examples; each value of $\lambda$ provides a different example. The generating function associated with each is

$$
F(x, \lambda ; \mu)=\sum_{i=0}^{\infty}\left[c_{i}(x) \lambda^{i}\right] \mu^{i}=f(x, \lambda \mu) .
$$

Our principal observations are that the numerical performance of the functional epsilon algorithm is poor qualitatively for values of $\lambda$ near $\lambda_{c}$. The generating function $F(x, \lambda ; \mu)$ has a pole at $\mu=\lambda / \lambda_{c}$ which is near unity. The following Figures 1a-1d display the functional Padé approximants of type [2/2] (i.e. the entry $\varepsilon_{2}^{(0)}(x)$ in the functional epsilon table) for the series generated by (a) $\lambda=2 / 3$, (b) $\lambda=0.74$, (c) $\lambda=0.7477$, (d) $\lambda=0.8$, and the approximants are labelled $B$ in each case. They are compared with the exact solution in each case, which is labelled $\mathrm{A}$, namely the analytic solution (3.8). We conclude that this approximation scheme is qualitatively unsatisfactory for cases when $\lambda \approx \lambda_{c}$, and we attribute the failure to the fact that (3.5) has unsatisfactory implications when the poles of the underlying generating function $F(x, \lambda ; \mu)$ lie near $\mu=1$. 


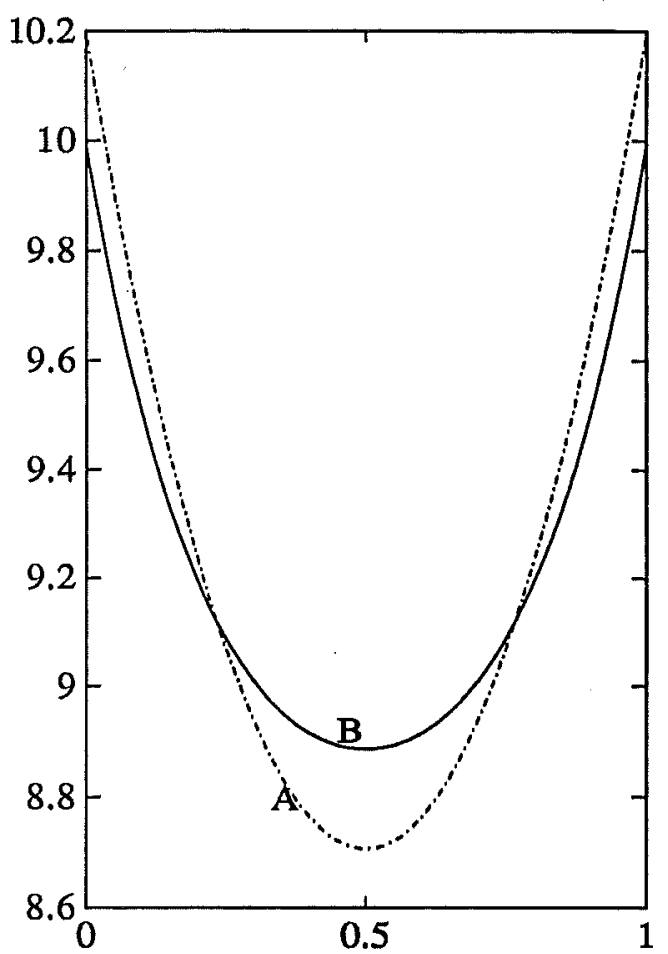

Fig 1a. $\lambda=\frac{2}{3}$

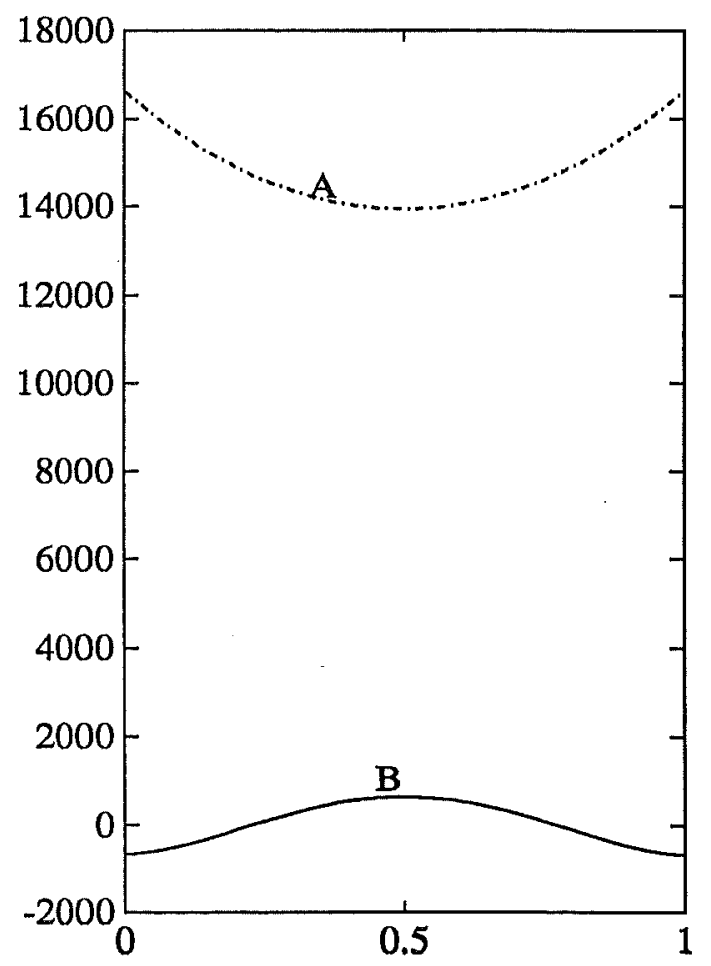

Fig 1c. $\lambda=0.7477$

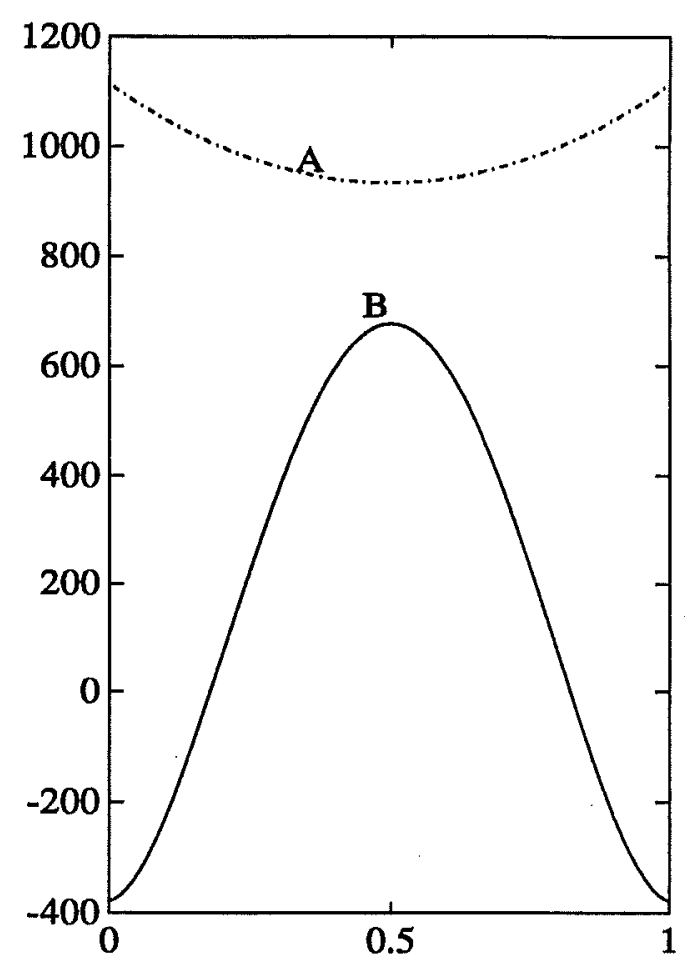

Fig 1b. $\lambda=0.747$

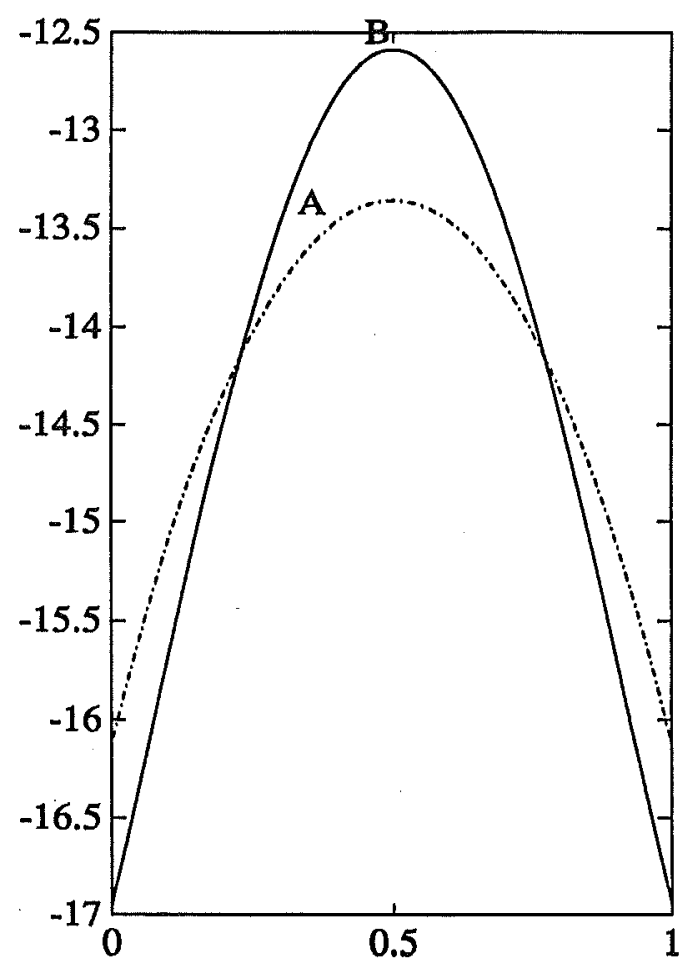

Fig 1d. $\lambda=0.8$

Figure 1 The curves show functional Padé approximants B against exact results $A$ for the example (3.6). 


\section{Hybrid vector Padé approximants}

The drawbacks and disadvantages of the vector epsilon algorithm, as compared with its scalar counterpart, stem from the fact that the limit of the underlying denominator polynomials is a polynomial which has roots of twice the multiplicity required. Hybrid vector Padé approximants are defined so that the denominator polynomials have the same degree as their scalar counterparts.

- The denominator polynomial of a hybrid vector Padé approximant is defined in terms of the roots $\zeta_{i}$ of the denominator of the corresponding vector Padé approximant. Suppose that $q(z)$ is the denominator of the vector Padé approximant of type $[n / 2 k]$. Because $q(z)$ is real analytic, we may express it as

$$
q(z)=\prod_{i=1}^{k}\left(z-\zeta_{i}\right)\left(z-\zeta_{i}^{*}\right)
$$

In the present case, in which $\zeta_{i}$ approximates a real pole position, the denominator polynomial $q^{H}(\lambda)$ of the corresponding hybrid vector Padé approximant is defined as

$$
q^{H}(z)=\prod_{i=1}^{k}\left(z-\operatorname{Re} \zeta_{i}\right)
$$

The associated numerator polynomial is defined as

$$
\boldsymbol{p}^{H}(z)=\left[\boldsymbol{f}(z) q^{H}(z)\right]_{0}^{n}
$$

Since $\partial\left\{q^{H}(z)\right\}=k, \partial\left\{\boldsymbol{p}^{H}(z)\right\}=n$, we call

$$
\boldsymbol{r}^{H}(z):=\boldsymbol{p}^{H}(z) / q^{H}(z)
$$

a hybrid vector Padé approximant of type $[n / k]$. We also recall that the entries of the modified Aitken $\Delta^{2}$ algorithm for accelerating the convergence of the vector sequence $S$ of (2.1) are

$$
\boldsymbol{t}_{n}:=\boldsymbol{s}_{n+1}-\Delta \boldsymbol{s}_{n+1} \frac{\left(\Delta \boldsymbol{s}_{n}\right)^{2}}{\Delta \boldsymbol{s}_{n} \cdot \Delta^{2} \boldsymbol{s}_{n}}, \quad n=0,1,2, \ldots
$$

and that the sequence $\left\{\boldsymbol{t}_{n}\right\}_{n=0}^{\infty}$ corresponds directly to the sequence of hybrid vector Padé approximants of type $[n / 1]$ to the generating function (2.9) evaluated at $z=1$, [Graves-Morris, 1992]. The functional form of (4.5) is

$$
t_{n}(x):=\sum_{i=0}^{n+1} c_{i}(x)-c_{n+2}(x) \frac{\int_{0}^{1}\left[c_{n+1}(x)\right]^{2} d x}{\int_{0}^{1} c_{n+1}(x) c_{n+2}(x) d x}
$$

expressed in the form appropriate for application to (3.7). 


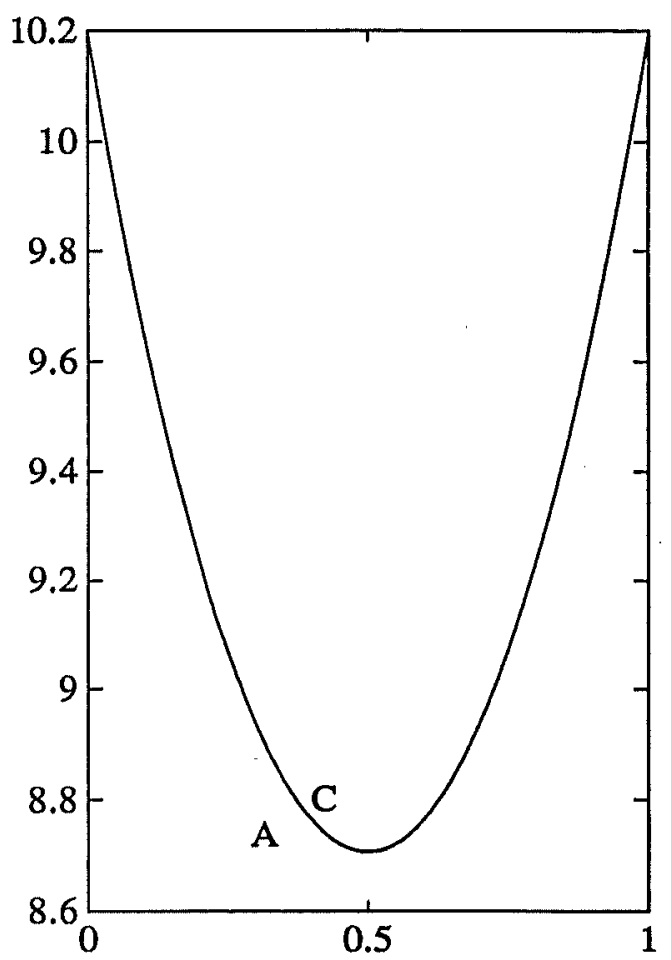

Fig 2a. $\lambda=\frac{2}{3}$

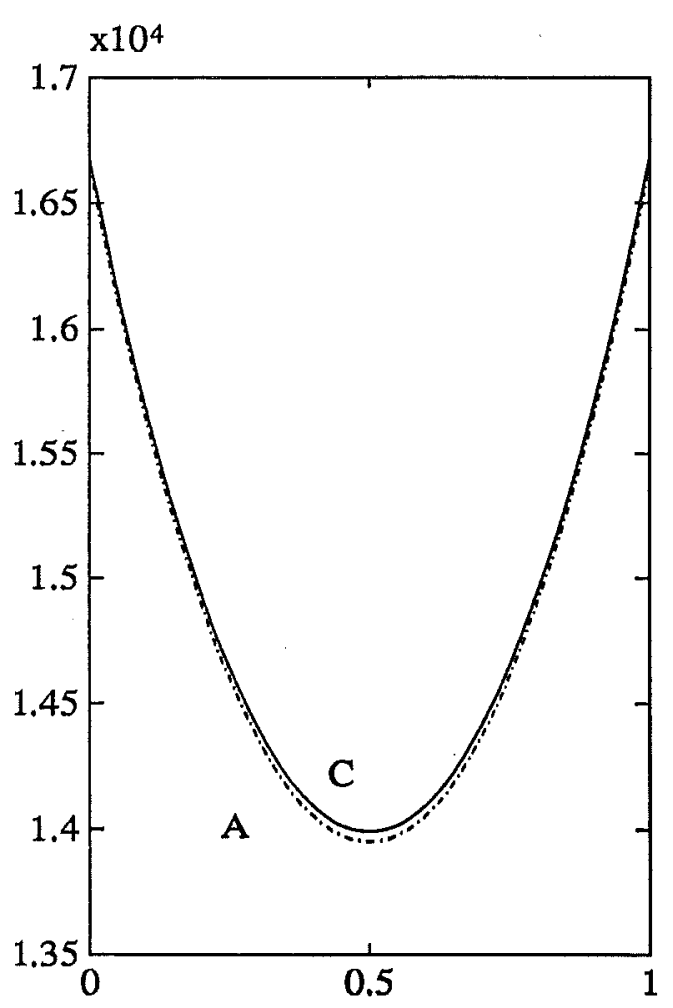

Fig 2c. $\lambda=0.7477$

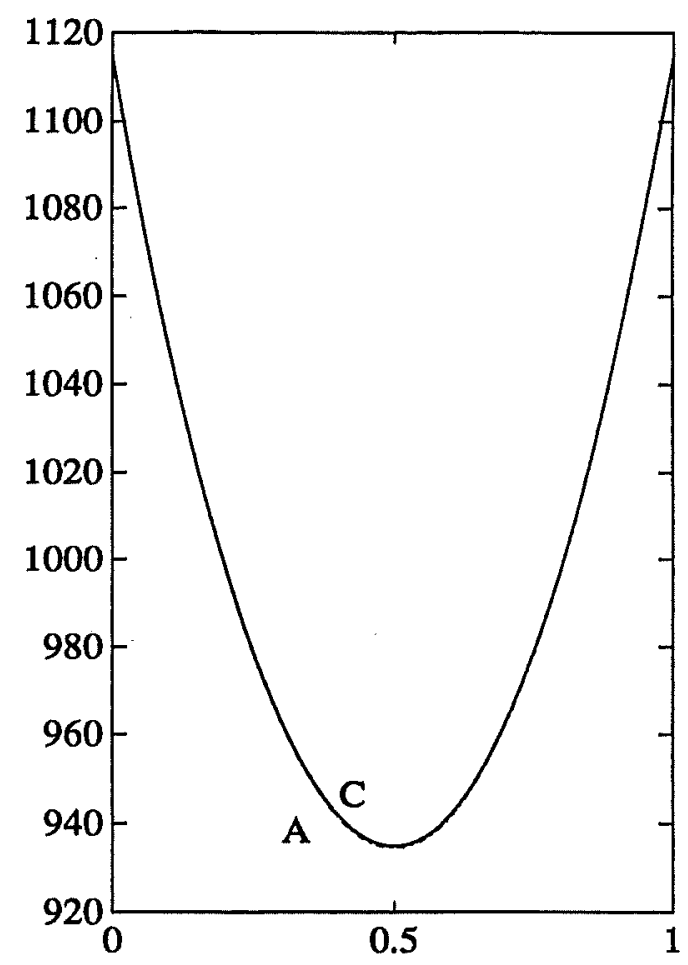

Fig 2b. $\lambda=0.747$

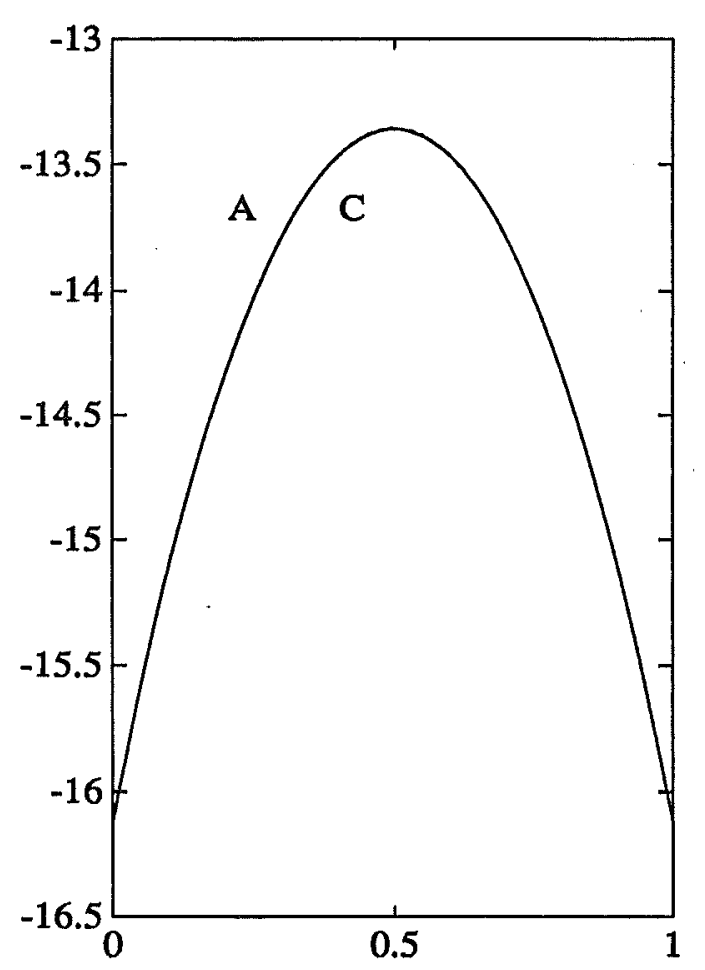

Fig 2d. $\lambda=0.8$

Figure 2 The curves show hybrid functional Padé approximants C against exact results $A$ for the example (3.6). 
We display the results of using this formula in Figures 2a-2d for the same values of $\lambda$, (a) $\lambda=2 / 3$, (b) $\lambda=0.74$, (c) $\lambda=0.7477$ and (d) $\lambda=0.8$.

The curves for $t_{n}(x)$ are labelled by $C$; the exact (analytic) results are also shown and again labelled by $\mathrm{A}$. Note that it is impossible to distinguish visually between the exact and the approximation results in Figures 2a and 2d. Accordingly, in Table 1 we display the approximation errors for all four cases considered. The columns labelled approximation error show the maximum relative approximation error over the range $0 \leq x \leq 1$, and quantify the results shown in Figures 1 and 2.

\begin{tabular}{|l|c|c|}
\hline \multirow{2}{*}{$\lambda$} & \multicolumn{2}{|c|}{ Approximation Error } \\
\cline { 2 - 3 } & VPA & Hybrid \\
\hline $2 / 3$ & 0.0207 & 0.0001 \\
0.74 & 0.3115 & 0.0001 \\
0.7477 & 1.0405 & 0.0030 \\
0.8 & 0.0577 & 0.0002 \\
\hline
\end{tabular}

Table 1. Maximum relative approximation errors.

A comparison of the results shown in Figures 1 and 2, or the results shown in Table 1, makes obvious the great superiority of the hybrid approximants in a numerical problem where the generating function has a nearby pole. The underlying analysis indicates that these conclusions are quite general.

\section{References}

[1] Baker, G.A. Jr. (1974): Essentials of Padé approximants. Academic Press, New York.

[2] Baker, G.A. Jr., Graves-Morris, P.R. (1981): Padé approximants. Addison Wesley, Cambridge.

[3] Brezinski, C. (1975): Généralisations de la transformation de Shanks, de la table de Wynn et de l' $\varepsilon$-algorithme. Calcolo 12, 317-360.

[4] Brezinski, C. (1980): Padé type approximation and general orthogonal polynomials. Birkhäuser Verlag.

[5] Graves-Morris, P.R. (1983): Vector-valued rational interpolants I. Numer. Math. 42, 331348. 
[6] Graves-Morris, P.R. (1990): Solution of integral equations using generalised inverse, function-valued Padé approximants I. J. Comput. Appl. Math. 32, 117-124.

[7] Graves-Morris, P.R. (1992): Extrapolation methods for vector sequences. Num. Math. 61, 475-487.

[8] Graves-Morris, P.R., Jenkins, C.D. (1986): Vector-valued rational interpolants III. Constr. Approx. 2, 263-289.

[9] Graves-Morris, P.R., Jenkins, C.D. (1989): Degeneracies of generalised inverse, vectorvalued Padé approximants. Constr. Approx. 5, 463-485.

[10] Graves-Morris, P.R., Saff, E.B. (1988): Row convergence theorems for generalised inverse, vector-valued Padé approximants. J. Comput. Appl. Math. 23, 63-85.

[11] McLeod, J.B. (1971): A note on the $\varepsilon$-algorithm. Computing 7, 17-24.

[12] Smith, D.A., Ford, W.F., Sidi, A. (1987): Extrapolation methods for vector sequences. SIAM Rev. 29, 199-233.

[13] Varga, R.S. (1962): Matrix iterative analysis. Prentice Hall, Englewood Cliffs, N.J.

[14] Wynn, P. (1962): Acceleration techniques for iterated vector and matrix problems. Math. Comput. 16, 301-322.

[15] Wynn, P. (1963): Continued fractions whose coefficients obey a non-commutative law of multiplication. Arch. Rat. Mech. Anal. 12, 273-312. 